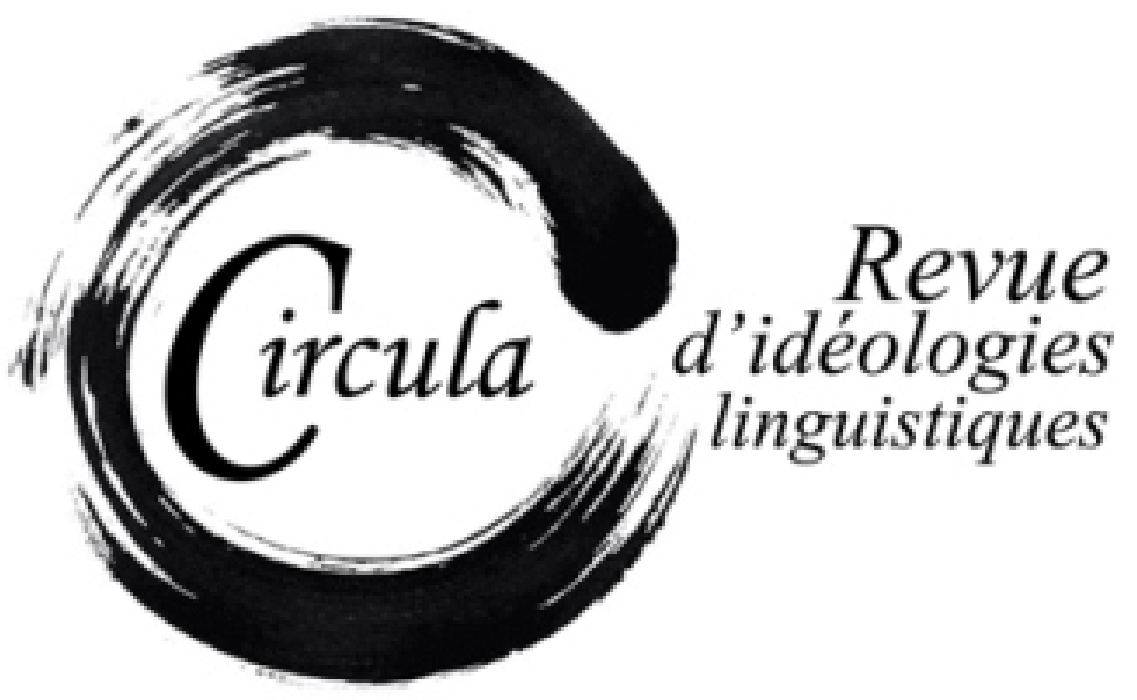

TITRE: L'ANGLICISME SYNTAXIQUE : PRODUIT INÉVITABLE DU CONTACT DES LANGUES?

Auteures: Shana Poplack, Université d'Ottawa, Nathalie Dion, Université d'OtTaWa et Lauren ZENTZ, UNIVERSITY OF HOUSTON

Revue: Circula, numéro 9 : A-t-on encore PeUR des anglicismes? PerCePtion actuelle des anglicismes au QuébeC ET DANS L'ESPACE FRANCOPHONE

PAGES: $77-106$

ISSN: 2369-6761

DiReCTRICES: MiREILlE ElChaCAR ET NADINE VINCENT

URI: HTTP://HDL.HANDLE.NET/11143/16048

DOI: HTTPS://DOI.ORG/10.17118/11143/16048 


\title{
L’anglicisme syntaxique : produit inévitable du contact des langues?
}

\author{
Shana Poplack, Université d'Ottawa \\ spoplack@uottawa.ca
}

Nathalie Dion, Université d'Ottawa nathalie.dion@uottawa.ca

Lauren Zentz, University of Houston

Irzentz@ central.uh.edu

\begin{abstract}
Résumé : Il est communément admis que le contact linguistique provoque la convergence grammaticale. L'objectif de cette recherche est de proposer et d'appliquer une méthode empirique pour vérifier cette hypothèse. Nous illustrons l'utilité de cette méthode par l'entremise du cas des prépositions sans régime en français, trait stéréotypé attribué à l'influence de l'anglais. Nos résultats, révèlent plusieurs preuves contrecarrant cette interprétation. En revanche, elles démontrent que le candidat à la convergence est plutôt survenu par l'extension d'une tournure purement française à un nouveau contexte. Nous concluons que le changement causé par le contact n'est pas donné d’emblée ; il doit être soigneusement établi par une méthodologie scientifique telle que proposée ici.
\end{abstract}

Mots-clés : anglicismes ; convergence grammaticale ; contact linguistique ; variabilité linguistique ; propositions nues

\begin{abstract}
That language contact results in linguistic change is a widespread assumption, but enjoys relatively little empirical support. In this paper we propose and test a scientific method to determine the effects that contact languages exert on each other. We illustrate its utility with preposition stranding in North American French, widely believed to result from centuries of intense contact with English. Systematic analyses provide no evidence of convergence, revealing instead that the offending construction arose from analogical extension of a native French construction to a novel context. We conclude that grammatical convergence must be proved on a case-by-case basis, using methodology capable of distinguishing internal from contact-induced change.
\end{abstract}

Keywords: anglicisms; grammatical convergence; language contact; linguistic variation; bare prepositions 


\section{Introduction ${ }^{1}$}

Devrait-on avoir peur des anglicismes? Nombreux sont ceux qui diraient oui, surtout au Canada. Quarante ans après le passage de la Loi 101, qui a fait du français la langue officielle du Québec, et la revendication de cette langue par les francophones partout au Canada, l'influence de l'anglais sur le français demeure une préoccupation majeure². L'ampleur de cette préoccupation nous est récemment redevenue apparente grâce à l'afflux massif de réactions qu'a provoquées un communiqué de presse (Université d'Ottawa, 2018) présentant les grandes lignes de nos recherches sur les anglicismes lexicaux (Poplack, 2018 ; Poplack et Dion, 2012). Près de 1000 commentaires sur Facebook, Twitter et Reddit en réponse au communiqué témoignent du vif intérêt que porte le public à cette question. Maintes inquiétudes ont été exprimées, mais les principales concernent l'appauvrissement de la langue, une diminution de sa qualité, et même sa disparition totale.

L'idée selon laquelle le contact linguistique entraîne inévitablement de tels changements à l'une ou aux deux langues impliquées n'est certes pas limitée au contexte canadien ni au contact entre le français et l'anglais. Il se reproduit presque partout où le bilinguisme est répandu. En tant que linguistes, de telles convictions nous intéressent de façon particulière, puisqu'en réalité, on en connaît très peu sur les mécanismes qui sous-tendent le changement causé par le contact. On s'interroge toujours sur le niveau d'intensité et la durée du contact requis pour provoquer de tels changements, ainsi que sur la nature précise des processus impliqués. Bien qu'il s'agisse de questions d'ordre sociolinguistique, elles ont rarement fait l'objet d'analyses empiriques systématiques. Par conséquent, au Laboratoire de sociolinguistique de l'Université d'Ottawa, nous nous efforçons de combler ces lacunes, dans le but de vérifier le bien-fondé de ces idées reçues au moyen d’analyses scientifiques à grande échelle. Sachant que la langue parlée au quotidien est le lieu privilégié du contact et du changement linguistiques, c'est elle que nous ciblons comme source de données. Dans ce qui suit, nous détaillons les méthodes que nous avons déployées pour effectuer de telles analyses et nous présentons les résultats surprenants qui en découlent quand on les applique à un candidat paradigmatique du statut d'« anglicisme».

\subsection{Qu'est-ce que l'anglicisme?}

N'étant pas un terme technique, il convient d'expliquer ce que nous entendons par « anglicisme » avant d'aller plus loin. L'influence éprouvée par une langue - appelons-la la langue receveuse en contact avec une autre (langue donneuse), revêt trois formes principales dans les interactions spontanées bilingues (Poplack, 2015, 2018). L'une implique l'insertion de mots isolés d'une langue

1. La recherche présentée dans ce chapitre a bénéficié de l'appui généreux du Conseil de recherches en sciences humaines du Canada. Poplack détient la Chaire de recherche du Canada (I) en linguistique. Nous remercions Sabine Huynh pour son aide précieuse avec la formulation française de certaines sections de ce chapitre.

2. Les minorités anglophones au Québec estiment inversement que le contact avec le français a mené à l'appauvrissement de leur anglais (Poplack, Walker et Malcolmson, 2006). 
donneuse (l'anglais dans l'exemple illustré en (1)) dans un énoncé autrement produit en la langue receveuse. Il s'agit là de l'emprunt lexical. Une deuxième consiste en la juxtaposition de séquences de mots provenant d'une langue et de l'autre, communément appelée l'« alternance de code », tel qu'en (2). Enfin, la langue donneuse peut imposer sa structure grammaticale à la langue receveuse, souvent sans aucun indice visible. Nous qualifions ce processus, parfois appelé « anglicisme syntaxique », de « convergence grammaticale» (3).

(1) Il va dire, « vous voulez avoir une bière, un café ? » Tu sais, les entertainer ['accueillir'], tu sais. $(\mathrm{OH} .040 .1545)^{3}$

(2) Puis ça c'est toute bien pas trop de sa faute à lui. He just can't do it ['il ne peut simplement pas le faire'], vois-tu? Tu sais, il est pas capable. Fait que you have to ['c'est toi qui dois le faire']. (OH.007.1426)

(3) C'est $30^{\circ}$ en ce moment. (Delisle, 1998: 10)

L'analyse quantitative systématique d'emprunts lexicaux d'origine anglaise en français (et d'origine française en anglais, ainsi qu'à travers plusieurs autres paires de langues) a démontré 1) qu'ils sont très rares, 2) qu'ils sont éphémères, et 3) qu'ils revêtent rapidement la grammaire de la langue receveuse (Poplack, 2017, 2018 ; Poplack et Dion, 2012, entre autres). De par ces faits mêmes, ils ne peuvent donc pas changer la structure de la langue receveuse. De même, les séquences juxtaposées qui constituent les alternances de code se conforment à la grammaire respective de leur langue d'origine : les portions françaises portent la morphologie et obéissent à la syntaxe du français, alors que les contreparties anglaises s'alignent sur cette langue (p. ex. Poplack, 1980 ; Poplack et Dion, 2012). Aucune des langues n'infiltre l'autre, et ce, même dans des cas où l'alternance a lieu à l'intérieur d'une seule phrase, tels ceux en (4).

(4) a. Il a dit que des fois, là, quand il marchait, là, il marchait over dead bodies ['sur des cadavres']. (OH.014.746)

b. Si tu es pas capable de les battre, join them ['joins-toi à eux']. (OH.048.2730)

La principale question par rapport aux alternances de code concerne plutôt leur positionnement, et les analyses quantitatives confirment que celui-ci est limité à un nombre restreint de sites spécifiques où les grammaires des deux langues impliquées se chevauchent (Poplack, 1980, 1985, 2015 ; Sankoff et Poplack 1981, entre autres). Puisque les contextes qui admettent les alternances de code respectent simultanément les deux grammaires en présence au lieu d'enfreindre l'une ou l'autre, il s'ensuit que cette stratégie ne peut pas changer la structure des langues en contact elle non plus.

3. Les codes entre parenthèses font référence aux numéro de locuteur et numéro de ligne dans la transcription des Corpus du français parlé à Ottawa-Hull (OH ; Poplack, 1989a), Corpus de l'anglais parlé au Québec (QEC ; Poplack, Walker et Malcolmson, 2006) ou Récits du français québécois d'autrefois (RFQ ; Poplack et St-Amand, 2009). Tous les exemples, standards ou non, sont reproduits fidèlement dans leur version originale à partir des énoncés des locuteurs. 
Les résultats esquissés ci-dessus sont très robustes, ayant été reproduits sur plus de 13 paires de langues et des dizaines de milliers d'occurrences (Budzhak-Jones, 1998 ; Ghafar Samar et Meechan, 1998 ; Moinzadeh, 1999 ; Mustafawi, 2002 et Poplack, 2018 entre autres). Néanmoins, beaucoup de parties concernées demeurent sceptiques quant à la capacité de la langue receveuse à résister aux incursions de la langue donneuse. Ce qu'elles craignent d'abord et avant tout est l'insidieuse convergence grammaticale, comme l'a si bien exprimé cet individu anonyme en réponse à un des articles publiés par Radio-Canada (2018):

(5) Je suis d'accord que le franglais, ou l'alternance des codes, sur le plan lexical n'est pas très dangereux. [...] Shana Poplack n'a pas parlé des anglicismes syntaxiques et sémantiques. Ces anglicismes sont beaucoup plus insidieux, car ils représentent des structures ou pensées anglaises. Quand on dit «faire du sens » ou « prendre pour acquis », on est en train de penser en anglais. Il en va de même pour le mot « bienvenue » dans le sens de « il n’y a pas de quoi ». À mon avis, ce genre de franglais représente assurément un danger pour la langue française, car l'anglicisme est camouflé dans des mots français, et sans le savoir, le locuteur de ces énoncés pense en anglais.

En fait, il est communément admis que la convergence grammaticale est le résultat naturel du contact de langues (p. ex. Appel et Muysken, 1987 : 154 ; Backus, 2004, 2005 ; Harris et Campbell, 1995 : 149 ; Toribio, 2004 ; Winford, 2005). Armées des outils de la sociolinguistique variationniste, dans cet article nous proposons et testons une méthode capable de trancher si une caractéristique grammaticale stéréotypée et stigmatisée du français québécois s'est réellement développée suite au contact intense et de longue durée avec l'anglais. Notre candidat à la convergence est le placement variable de la préposition dans les subordonnées relatives déterminatives, tel qu'illustré en (6), où les locuteurs placent tantôt la préposition à la tête de la proposition, accompagnée du reste du syntagme prépositionnel (6a), tantôt à la fin, sans régime adjacent (6b).

(6) a. Les anglaises avec qui je parlais, ils le croyaient pas. (OH.082.1695)

b. J'avais pas personne à parler avec. (OH.013.1964)

Alors que la norme interdit le placement des prépositions à la fin d'un syntagme dans les deux langues, cette configuration représente la norme en anglais (7), langue avec laquelle le français est en contact intense au Canada depuis des siècles.

(7) This is something that I could probably get interested in. (QEC.301.1372)

'Ceci est quelque chose que je pourrais probablement devenir intéressé dedans.'

Un des objectifs de cet article est de déterminer si l'emplacement de avec en fin de syntagme en français (6b) est le résultat de ce contact, ou s'il est attribuable - malgré les ressemblances superficielles avec les prépositions échouées de l'anglais - à des facteurs tout autres. Poplack et Levey 
(2011) proposent plusieurs tests concrets de la convergence, dont la plupart comportent des comparaisons avec des variétés-repère (v. aussi Torres Cacoullos et Travis, 2018). Afin de conclure qu'il y a eu un changement causé spécifiquement par le contact, il faudra démontrer que la construction examinée $1^{\circ}$ ) est effectivement le résultat d'un changement ; $2^{\circ}$ ) n'était pas présente dans la langue avant le contact ; $3^{\circ}$ ) n'est pas présente dans une variété contemporaine qui est à l'abri du contact ; $4^{\circ}$ ) se comporte de la même manière que son équivalent (c'est-à-dire la forme qui aurait été empruntée) dans la variété donneuse ; et $5^{\circ}$ ) diffère sensiblement d'une structure semblable dans la langue receveuse, s'il y en a une ${ }^{4}$. Dans cet article, nous abordons chacun de ces critères, en nous concentrant en particulier sur $4^{\circ}$ et $5^{\circ}$. Par le biais de comparaisons quantitatives systématiques, nous situons les prépositions de fin de syntagme par rapport, d'une part, à leurs homologues apparents en anglais, et d'autre part, aux options de souche française de placement des prépositions.

\subsection{Le placement variable de la préposition dans les propositions relatives françaises}

Bien que la règle normative du français exige en général que la préposition soit suivie immédiatement de son régime (Grevisse et Goosse, 2016: 1441), les prépositions sans régime sont aussi admises dans certains contextes spécifiques, comme nous le verrons en détail dans la section 6 . Toutefois, les propositions relatives ne figurent pas parmi eux. Voilà sans doute pourquoi les exemples comme (6b) ont attiré tant d'attention en français nord-américain (p. ex. Flikeid, 1989; King, 2000 ; King et Roberge, 1990 ; Roberge et Rosen, 1999 ; Roy, Lefebvre et Régimbald, 1982 ; Vinet, 1984). En effet, leur apparente absence dans d'autres langues romanes et d'autres variétés du français (Vinet, 1979 : 117, 1984 : 234) en a mené plusieurs à conclure que la présence de telles prépositions «échouées » est un changement attribuable au contact avec l'anglais en Amérique du Nord. Or, puisque la convergence naît de l'usage spontané, nous nous proposons de vérifier, dans le parler français de tous les jours, si le choix de placement de la préposition suit un schéma anglais ou français.

En outre, les exemples précédents montrent que le placement de la préposition est un processus variable. Il faudra donc contextualiser la variante échouée (ÉCH. ; [6b], [8]) par rapport aux autres possibilités de placement auxquelles elle fait la concurrence. Celles-ci comprennent l'antéposition (ANT.) exemplifiée en (6a) et (9), ainsi que l'élimination complète de la préposition, à travers un processus connu comme l'« absorption» (ABS. ; Barbaud [1998] ; voir aussi Bouchard [1982], Frei [1929/1971], Roberge et Rosen [1999] et Vinet [1984]), illustrée en (10). Pour en arriver à une description adéquate de la trajectoire de la préposition échouée en français, il faut d'abord établir comment cette variante interagit avec ces options de souche française, et déterminer ce qui motive le choix de l'une plutôt que des autres. Nous porterons notre attention sur ces questions dans les sections qui suivent.

4. Certains chercheurs (p. ex., Heine et Kuteva, 2005) soutiendraient que le critère $4^{\circ}$ n'est pas strictement obligatoire, puisque le contact pourrait simplement déclencher un développement interne. En l'absence d'un test clair de cette hypothèse, nous continuons toutefois d'invoquer une comparaison systématique entre le candidat à la convergence et la source présumée de l'emprunt. 
(8) Ça c'est un gars que j'ai déjà sorti avec $[$ [ÉcH.]. $(O H .090 .1170)$

(9) Je protégeais le monde avec qui ${ }_{\text {[ANT.] }}$ je traitais. $(\mathrm{OH} .082 .2947)$

(10) Ils m’ont donné là, disons le nécessaire là, ce que j’avais besoin [ ] [ABS.] $(\mathrm{OH} .111 .421)$

\section{Données et méthodologie}

\subsection{La situation de contact}

Les données linguistiques sur lesquelles nous basons cette étude ont été recueillies le long de la frontière entre le Québec et l'Ontario, dans la région de la capitale fédérale du Canada. II s'agit du site d'un contact intense entre le français, langue officielle et majoritaire du Québec, et l'anglais, son homologue dans la province de l'Ontario. L'analyse repose sur un corpus informatisé construit à partir de conversations spontanées enregistrées auprès d'un échantillon prélevé au hasard de 120 francophones bilingues habitant dans cette région (Poplack, 1989a, b).

\subsection{L'application du cadre théorique variationniste comparatif à l'examen de la convergence grammaticale}

L'approche variationniste aborde l'étude du langage sous l'optique des choix que font sans cesse les locuteurs, au cours de leurs interactions quotidiennes, parmi différentes options qui ont une même signification ou fonction référentielle dans des contextes linguistiques précis. Ces choix ne sont pas tenus comme libres, mais sont plutôt sujets à des contraintes imposées par les caractéristiques de l'environnement linguistique et extralinguistique dans lequel ils se produisent. Ces caractéristiques sont opérationnalisées en tant que facteurs représentant des hypothèses sur ce qui motive le choix d'une variante plutôt que d'une autre. Parmi les hypothèses que nous examinons ici, certaines touchent aux caractéristiques de la préposition elle-même (fonction, poids sémantique, identité lexicale), d'autres se rapportent au verbe dont elle est le complément (besoin d'un syntagme prépositionnel explicite afin d'interpréter les nuances sémantiques du verbe, identité lexicale), et d'autres encore s'appliquent au régime (structure, animéité, adjacence au verbe). Chacune de ces hypothèses a déjà été invoquée en rapport avec le choix de l'une des trois stratégies de placement de la préposition ${ }^{5}$ (Ambrose, 1987 ; Bouchard, 1982 ; Grevisse et Goosse, 2016 ; Kayne, 1975 ; Koster, 1978 ; Takami, 1992 ; Vinet, 1984 ; Zribi-Hertz, 1984).

5. Pour de plus amples détails sur les facteurs analysés et les hypothèses afférentes, voir Poplack, Zentz et Dion (2012). 


\subsection{Démarche}

Nous avons extrait chaque proposition relative déterminative à complément prépositionnel avec ou sans régime exprimé (en d'autres mots, le « contexte variable » [Labov, 1969 : 728-729] de l'échouage) dans les conversations enregistrées avec les locuteurs qui constituent notre échantillon. Nous les avons codées selon tous les facteurs énumérés précédemment. Des analyses multivariées (Sankoff, 1988 ; Sankoff, Tagliamonte et Smith, 2005) indépendantes nous ont permis de déterminer les facteurs qui produisent des effets statistiquement significatifs sur le choix de la stratégie de placement de la préposition, ainsi que la direction et l'ampleur relatives de ces effets. Nous interprétons les hiérarchies des contraintes produites par ces analyses comme la structure du mécanisme de sélection, et nous nous servons de ces renseignements pour déterminer l'origine des prépositions sans régime dans les propositions relatives. En comparant cette structure variable parmi les différentes variétés-repère dont nous disposons, nous évaluerons si un changement a réellement eu lieu, et le cas échéant, sa nature.

\section{Analyse de la variabilité}

\subsection{Distribution des variantes}

Notre première découverte a été que les contextes qui admettent l'échouage (les propositions relatives déterminatives) sont dans l'ensemble très rares. Seuls 340 des milliers de syntagmes nominaux (sujet et objet) tirés de nos données qui auraient pu être relativisés le sont. Et dans ce contexte déjà peu fréquent, les prépositions ne sont échouées que dans un maigre $12 \%$ des cas (Tableau 1).

Tableau 1. Répartition globale des stratégies de placement de la préposition dans propositions relatives

\begin{tabular}{|l|c|c|}
\hline Variantes & \% & N \\
\hline Absorption & 51 & 172 \\
\hline Antéposition & 37 & 127 \\
\hline Échouage & 12 & 41 \\
\hline Total & 100 & 340 \\
\hline
\end{tabular}

La saillance de la préposition échouée dans l'imaginaire collectif dépasse donc de loin son importance dans l'usage réel. La variante prescrite - la préposition antéposée - qui à elle seule représente $37 \%$ des données, est trois fois plus fréquente que la préposition échouée. La stratégie privilégiée est en fait l'absorption. La préposition échouée n'est donc qu'une variante parmi d'autres - la plus rare en plus. Son emploi est le fruit d'un choix effectué par les locuteurs. Qu'est-ce qui motive ce choix? Est-ce l'influence de l'anglais, comme plusieurs le prétendent? Pour répondre, il faut savoir 
comment elle interagit avec les autres variantes, ce que révèlent les analyses du conditionnement de la variation présentées dans les prochaines sections.

\subsection{Conditionnement de la variation}

\subsubsection{L'échouage}

L'analyse multivariée des facteurs susceptibles de contribuer à la sélection de l'échouage, opérationnalisés à partir des hypothèses décrites ci-dessus (Tableau 2), montre que le meilleur prédicteur (probabilité de .99) est de loin le poids sémantique de la préposition6. Tel qu'observé ailleurs (Ambrose, 1987 ; Bouchard, 1982 ; Koster, 1978 ; Takami, 1992 ; Vinet, 1984 ; Zribi-Hertz, 1984), les prépositions sémantiquement fortes (c.-à-d. porteuses de contenu sémantique) favorisent nettement l'échouage. Les prépositions faibles ne paraissent, quant à elles, presque jamais sans régime?.

\section{Tableau 2. Analyse multivariée de la contribution de facteurs au choix de l'échouage (seuls} les facteurs statistiquement significatifs sont montrés)

\begin{tabular}{|c|c|c|c|}
\hline N total d'occurrences: & \multicolumn{3}{|c|}{$41 / 340$} \\
\hline \multirow[t]{2}{*}{ Tendance globale: } & \multicolumn{3}{|c|}{.12} \\
\hline & Probabilité & $\%$ & $\mathbf{N}$ \\
\hline \multicolumn{4}{|c|}{ POIDS SÉMANTIQUE DE LA PRÉPOSITION } \\
\hline Fort & .99 & 50 & $39 / 78$ \\
\hline Faible & .21 & 1 & $2 / 262$ \\
\hline Portée & 78 & & \\
\hline \multicolumn{4}{|l|}{ STRUCTURE DU RÉGIME } \\
\hline Pseudo-clivée & .91 & 18 & $7 / 39$ \\
\hline Relative ordinaire & .43 & 13 & $29 / 217$ \\
\hline Clivée & .42 & 6 & $5 / 84$ \\
\hline Portée & 49 & & \\
\hline \multicolumn{4}{|c|}{ PRÉSENCE D'UN AUTRE COMPLÉMENT } \\
\hline Aucun & .62 & 16 & $33 / 203$ \\
\hline Clitique & .49 & 8 & $5 / 62$ \\
\hline Post verbal & .22 & 4 & $3 / 75$ \\
\hline Portée & 40 & & \\
\hline
\end{tabular}

6. L'importance relative de l'effet d'un facteur, ici et dans les tableaux qui suivent, est mesurée par sa portée : la différence entre les poids des facteurs les plus et moins élevés dans un groupe de facteurs. Dans le Tableau 2, la portée du facteur du poids sémantique est de 78.

7. Pour opérationnaliser la notion de poids sémantique, nous avons distingué les prépositions dont le sens ne pouvait être décodé que par le contexte (p. ex. parler de, venir de), que nous avons codé comme « faibles », de celles dont l'interprétation est indépendante du contexte ( fortes »). 
Ceci dit, en regardant de plus près (Tableau 3), nous découvrons que les étiquettes « forte » et «faible » masquent un effet lexical idiosyncrasique : 94 \% (247/262) de toutes les prépositions classées comme faibles sont en réalité des occurrences de à et de, qui elles n’apparaissent pratiquement jamais à la fin d'un syntagme (2/39). De même, avec constitue la moitié (39/78) de la catégorie des prépositions fortes, qui à leur tour représentent à elles seules plus des deux tiers (25/39) des prépositions échouées. En fait, l'échouage est si restreint au niveau lexical que 90 \% (35/39) des cas sont constitués de quatre prépositions (toutes fortes) : avec, pour, dedans et dessus ${ }^{8}$.

\section{Tableau 3. Taux d'échouage selon l'identité lexicale et le poids sémantique de la préposition}

\begin{tabular}{|l|c|c|c|c|}
\hline \multirow{2}{*}{} & \multicolumn{4}{|c|}{ Poids sémantique } \\
\cline { 2 - 5 } & \multicolumn{2}{|c|}{ Faible } & \multicolumn{2}{c|}{ Fort } \\
\hline Identité lexicale & $\%$ & $\mathbf{N}$ & $\mathbf{N}$ \\
\hline$\dot{a}$ & 0 & $0 / 159$ & 0 & $0 / 1$ \\
\hline de & 2 & $2 / 88$ & 0 & $0 / 2$ \\
\hline avec & - & - & 64 & $25 / 39$ \\
\hline dedans & - & - & 75 & $3 / 4$ \\
\hline pour & - & - & 60 & $3 / 5$ \\
\hline dessus & - & - & 100 & $4 / 4$ \\
\hline Autres prépositions & 0 & $0 / 15$ & 17 & $4 / 23$ \\
\hline Total & 1 & $2 / 262$ & 50 & $39 / 78$ \\
\hline
\end{tabular}

\subsubsection{L'absorption}

Nous avons noté à la section 3.1 que les prépositions ne sont échouées que dans $12 \%$ des contextes admissibles. Qu'en est-il des 88 \% restants des données? Plus de la moitié sont absorbées (Tableau 1), faisant de l'absorption la variante prédominante. II faut noter que cette forme n'a pas de véritable équivalent en anglais (11).

(11) Les fêtes qu'on allait [ ] [ABS.] ça durait plus longtemps. (OH.002.976)

*'The parties that we went [ ], they lasted longer.'

L'absorption a été analysée comme une tentative d'éviter l'échouage qui découle du remplacement continu en langue parlée des pronoms relatifs porteurs du contenu sémantique d'une préposition (par ex., dont, duquel) par que, qui est moins riche sur le plan sémantique (Roberge et Rosen, 1999). La présence de que fait de la position postverbale l'unique emplacement disponible pour la préposition, que les locuteurs préféreraient (apparemment) éliminer qu'échouer.

8. Le fait que l'emploi de dedans et dessus en tant que prépositions soit critiqué par les instances normatives n'empêche pas qu'elles sont bel et bien employées comme telles dans la langue parlée, comme le témoigne l'exemple (i) :

(i) Puis il y a pas de statique dedans ça, c’est du verre étiré. (OH.082.254) 
Tableau 4. Analyse multivariée de la contribution de facteurs au choix de l'absorption (seuls les facteurs statistiquement significatifs sont montrés)

\begin{tabular}{|c|c|c|c|}
\hline N total d'occurrences: & \multicolumn{3}{|c|}{$172 / 340$} \\
\hline \multirow[t]{2}{*}{ Tendance globale: } & \multicolumn{3}{|c|}{.49} \\
\hline & Probabilité & $\%$ & $\mathbf{N}$ \\
\hline \multicolumn{4}{|c|}{ POIDS SÉMANTIQUE DE LA PRÉPOSITION } \\
\hline Faible & .62 & 60 & $157 / 262$ \\
\hline Forte & .17 & 19 & $15 / 78$ \\
\hline Portée & 45 & & \\
\hline \multicolumn{4}{|l|}{ STRUCTURE DU RÉGIME } \\
\hline Clivée & .62 & 62 & $52 / 84$ \\
\hline Relative ordinaire & .54 & 53 & $114 / 217$ \\
\hline Pseudo-clivée & .12 & 15 & $6 / 39$ \\
\hline Portée & 40 & & \\
\hline
\end{tabular}

L'analyse multivariée reproduite dans le Tableau 4 révèle que deux facteurs contribuent de façon sensiblement égale (comme l'indique leur portée) au choix de l'absorption : la structure du régime, où les pseudo-clivées la défavorisent, et le poids sémantique de la préposition. Ici, ce sont les prépositions faibles qui encouragent l'absorption, au contraire de ce que nous avions trouvé pour l'échouage. En fait, 91 \% (157/172) des prépositions absorbées sont faibles. On retrouve aussi le même effet lexical prépondérant : à quelques exceptions près, il s'agit surtout de à et de. Ces prépositions absorbées sont précisément celles qui, comme nous l'avons vu, ne sont pratiquement jamais échouées.

\subsubsection{L'antéposition}

La troisième possibilité de placement de la préposition est l'antéposition (6a et 9). Cettevariante, qui d'ailleurs est la seule qui est prescrite, attire bien moins d'attention que les deux autres. Néanmoins, elle est aussi très robuste, et représente plus d'un tiers des données (Tableau 1). Le Tableau 5 affiche les facteurs qui régissent sa sélection. 
Tableau 5. Analyse multivariée de la contribution de facteurs au choix de l'antéposition (seuls les facteurs statistiquement significatifs sont montrés)

\begin{tabular}{|c|c|c|c|}
\hline N total d'occurrences: & \multicolumn{3}{|c|}{$127 / 340$} \\
\hline \multirow[t]{2}{*}{ Tendance globale: } & \multicolumn{3}{|c|}{.37} \\
\hline & Probabilité & $\%$ & $\mathbf{N}$ \\
\hline \multicolumn{4}{|l|}{ STRUCTURE DU RÉGIME } \\
\hline Pseudo-clivée & .78 & 67 & $26 / 39$ \\
\hline Relative ordinaire & .48 & 34 & $74 / 217$ \\
\hline Clivée & .41 & 32 & $27 / 84$ \\
\hline Portée & 37 & & \\
\hline \multicolumn{4}{|c|}{ PRÉSENCE D'UN AUTRE COMPLÉMENT } \\
\hline Post verbal & .68 & 51 & $38 / 75$ \\
\hline Clitique & .59 & 42 & $26 / 62$ \\
\hline Aucun & .41 & 31 & $63 / 203$ \\
\hline Portée & 27 & & \\
\hline
\end{tabular}

Ici aussi, la structure du régime exerce un effet statistiquement significatif : cette fois, les pseudo-clivées favorisent fortement l'antéposition, contrairement à ce que nous avons trouvé pour l'absorption. L’antéposition est également favorisée là où la préposition est séparée du verbe par un autre complément (postverbal ou clitique), et inhibée lorsqu'il n'y a pas d'autre régime ; ceci représente plutôt un contexte favorable à l'échouage. Mais le résultat le plus intéressant à nos fins est le fait que le poids sémantique n'a aucun effet sur l'antéposition. C'est la seule stratégie que nous avons examinée où ce facteur ne joue aucun rôle.

\subsubsection{Comparaison des stratégies de placement de la préposition}

Un examen minutieux des analyses précédentes suggère que les trois stratégies - l'échouage, l'absorption et l'antéposition - se divisent de concert le travail du placement de la préposition. Pour déterminer comment elles s'emboîtent dans les propositions relatives, nous avons réanalysé les données, en prenant en compte uniquement des facteurs sélectionnés comme statistiquement significatifs dans les Tableaux 2, 4 et 5 ci-dessus. 


\section{Tableau 6. Comparaison des facteurs contribuant à la sélection de la stratégie de placement de la préposition dans les propositions relatives}

\begin{tabular}{|c|c|c|c|}
\hline & Échouage & Absorption & Antéposition \\
\hline$N$ total d'occurrences: & $41 / 340$ & $172 / 340$ & $127 / 340$ \\
\hline Tendance globale: & .02 & .50 & .37 \\
\hline \multicolumn{4}{|c|}{ POIDS SÉMANTIQUE DE LA PRÉPOSITION } \\
\hline Forte & .98 & .18 & {[]$^{*}$} \\
\hline Faible & .24 & .61 & [ ] \\
\hline Portée & 74 & 43 & \\
\hline \multicolumn{4}{|c|}{ PRÉSENCE D'UN AUTRE COMPLÉMENT } \\
\hline Absence & .63 & [ ] & .44 \\
\hline Présence & .31 & [ ] & .59 \\
\hline Portée & 32 & & 15 \\
\hline STRUCTURE DU RÉGIME & [ ] & [ ] & [ ] \\
\hline
\end{tabular}

* Les crochets indiquent que le facteur en question n'exerce pas d'effet statistiquement significatif dans l'analyse en question.

Le schéma de distribution quasi complémentaire dévoilé dans le Tableau 6 révèle - remarquablement - que chaque variante joue un rôle exclusif dans le système. L'absorption sert à éliminer les prépositions faibles de la structure de surface. Ceci répond à la question de ce qu'il faut faire avec elles, puisqu'elles ne peuvent être échouées. L'antéposition assure l'analyse correcte de la préposition et du pronom relatif comme constituant unique ayant une fonction syntaxique interprétable. Ceci explique la préférence pour cette stratégie lorsqu'un complément du verbe supplémentaire est présent dans le syntagme. Situé par rapport à l'ensemble du système de placement de la préposition, le rôle de l'échouage devient clair : il est choisi le plus souvent dans des contextes où aucun élément n'intervient pour entraver l'interprétation du syntagme prépositionnel discontinu comme constituant unique. Cette stratégie n'est toutefois réservée qu'aux prépositions fortes.

\section{Comparaison avec la source présumée}

En gardant à l'esprit l'exigence selon laquelle le candidat à la convergence se comporterait, de façon non triviale, comme son équivalent dans la source présumée (Poplack et Levey, 2011 ; Thomason, 2001), nous examinons maintenant la structure du placement de la préposition dans une variété d'anglais canadien courant ${ }^{9}$ qui représente un modèle cible pour les locuteurs francophones bilingues dont nous avons étudié le comportement.

9. Il s'agit de l'anglais parlé dans les villes voisines largement monolingues d'Oshawa et de Whitby en Ontario (Poplack, Walker et Malcolmson, 2006). 


\subsection{Le placement de la préposition en anglais canadien courant}

Le Tableau 7 montre la répartition globale de l'antéposition, en (12), et l'échouage, illustré en (13) (et (7)), en anglais canadien courant.

Tableau 7. Répartition globale des stratégies de placement de la préposition dans les propositions relatives anglaises

\begin{tabular}{|l|c|c|}
\hline Variante & $\%$ & $\mathbf{N}$ \\
\hline Antéposition & 2 & 6 \\
\hline Échouage & 98 & 256 \\
\hline Total & 100 & 262 \\
\hline
\end{tabular}

(12) I think that- that the direction in which ${ }_{\text {[ANT.] }}$ Canadians want to go today is getting better. (QEC.317.746)

'Je crois que- que la direction dans laquelle les Canadiens veulent aller aujourd'hui s'améliore.'

(13) So- that's- pretty much all I could think of ${ }_{[\mathrm{E} C H .]}$ (QEC.046.806)

'C'est donc à peu près tout ce que je pouvais penser à.'

L'échouage, choisi dans $98 \%$ de toutes les propositions relatives, est clairement l'option par défaut; il est sans aucun doute assez robuste sur le plan quantitatif en anglais canadien pour fournir un modèle de placement de la préposition aux locuteurs francophones bilingues.

\section{Comparaison des stratégies de placement de la préposition entre langues en contact}

Afin d'appuyer la proposition selon laquelle les prépositions sans régime françaises ne sont pas le produit de la convergence avec les prépositions échouées de l'anglais, il nous faut démontrer qu'en dépit de leur ressemblance de surface, le système anglais de placement de la préposition diffère de celui qui opère en français. C'est sur cette question que nous nous penchons dans cette section.

Le point de départ de notre comparaison est la répartition globale des stratégies de placement dans les langues en contact. Les résultats présentés dans la Figure 1 illustrent de frappantes différences entre l'anglais et le français, non seulement en ce qui concerne le répertoire des variantes, mais aussi en ce qui a trait à leur répartition : bien que les prépositions soient presque catégoriquement échouées dans la première de ces langues, cette option est la moins fréquente dans la deuxième (12\%). L'antéposition représente plus d'un tiers (37\%) des données françaises, mais n'est choisie que dans $2 \%$ des cas en anglais. Et la variante principale en français, l'absorption (51\%), 
n'est même pas attestée en anglais. Rappelons aussi que l'anglais admet l'échouage dans plus de contextes syntaxiques (p. ex. les questions «QU », les constructions passives prépositionnelles) que le français, qui limite son usage à la subordonnée relative (Poplack, Zentz et Dion, 2012).

\section{Anglais canadien courant}

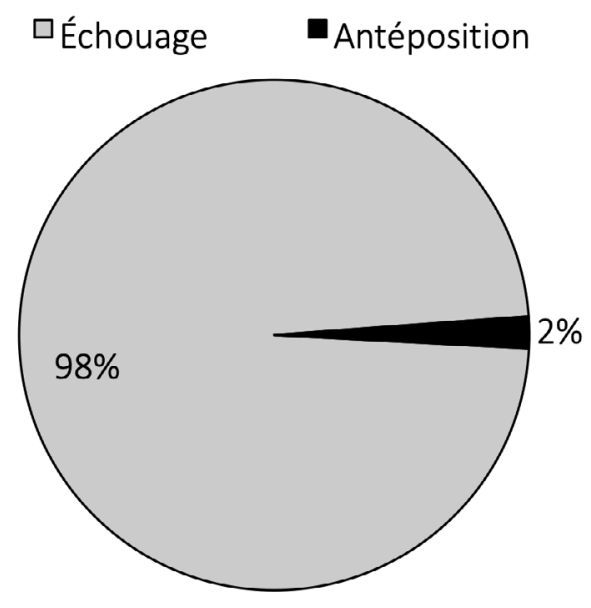

Français d'Ottawa-Hull

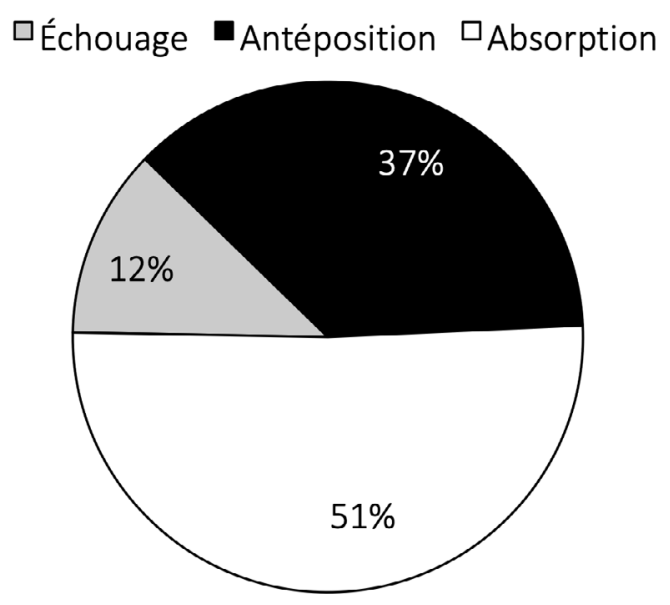

Figure 1. Comparaison de la répartition des variantes dans les langues en contact

Il y a donc peu de preuves convaincantes en faveur d'une analyse de convergence au niveau de la répartition des variantes. Mais la dominance écrasante de l'échouage en anglais pourrait tout de même avoir déclenché son infiltration encore embryonnaire en français. Le conditionnement de la variabilité, que nous interprétons comme la grammaire sous-tendant la sélection de la variante, est un meilleur indicateur. Nous comparons maintenant les contraintes régissant le placement variable de la préposition en français avec celles qui sont exploitées en anglais. Bien que l'énorme biais en faveur de l'échouage en anglais dilue forcément son conditionnement linguistique, nous mettrons néanmoins n'importe quels fragments de structure que nous décèlerions au profit cette comparaison.

\subsection{Conditionnement lexico-sémantique du choix de la variante}

En nous penchant d'abord sur l'identité lexicale du pronom relatif (Tableau 8), notons que tandis que l'antéposition est pratiquement restreinte à which 'que, dont' en anglais, elle se produit avec toute une gamme de pronoms en français. L'échouage, pour sa part, apparaît librement avec chaque pronom relatif en anglais ; en français, par contre, il se limite à que, $\varnothing$, à et pour, tel qu'illustré en (14). 
(14) a. Ça c'est le nom de celui que je reste avec ${ }_{[\mathrm{ÉcH.}]}(\mathrm{OH} .090 .1080)$

b. Puis il y a bien des affaires $\underline{\text { j}}$ 'avais de la misère avec $_{[\tilde{E} \mathrm{CH} .]}(\mathrm{OH} .052 .1216)$

c. Puis ceux-là qui disent toute ça, bien c'est un des premiers à allervoter pour $r_{[\hat{E} \mathrm{CH} .]} \cdot(\mathrm{OH} .003 .321)$

d. On avait rien pour jouer avec $[$ [ÉcH.] , hein? $(\mathrm{OH} .105 .643)$

Tableau 8. Répartition des pronoms relatifs dans les langues en contact selon la stratégie de placement de la préposition

\begin{tabular}{|l|c|c|c|c|c|c|c|}
\hline \multirow{2}{*}{} & \multicolumn{4}{|c|}{ Français } & \multicolumn{3}{c|}{ Anglais } \\
\cline { 2 - 8 } & abs. & éch. & ant. & & éch. & ant. & \\
\hline Pronom relatif & $\%$ & $\%$ & $\%$ & N & $\%$ & $\%$ & N \\
\hline que/that & 81 & 15 & 2 & 178 & 100 & 0 & 109 \\
\hline$\varnothing$ & 39 & 25 & 36 & 28 & 100 & 0 & 93 \\
\hline$\grave{a}$ & 86 & 14 & 0 & 14 & - & - & - \\
\hline pour & 44 & 56 & 0 & 9 & - & - & - \\
\hline où & - & - & 100 & 70 & - & - & - \\
\hline which & - & - & - & - & 58 & 42 & 12 \\
\hline who & - & - & - & - & 96 & 4 & 25 \\
\hline what & - & - & - & - & 100 & 0 & 25 \\
\hline Autres pronoms & 0 & 0 & 100 & 41 & - & - & - \\
\hline Total & 51 & 12 & 37 & 340 & 98 & 2 & 264 \\
\hline
\end{tabular}

L'identité lexicale de la préposition joue aussi un rôle dans le choix de la variante (Tableau 9). En anglais tout comme en français, les données sont dominées par un nombre limité de prépositions. Quatre d'entre elles (with 'avec', to 'à', about 'de' et for 'pour') représentent deux tiers (175/260) des données anglaises. Elles sont toutes extrêmement propices à l'échouage, mais les prépositions moins fréquentes le sont aussi. En français, avec, à et de représentent à elles seules 85 \% de toutes les prépositions (289/340). Mais en contraste frappant avec l'anglais, seule avec participe librement à l'échouage. 
Tableau 9. Taux d'échouage dans les langues en contact selon l'identité lexicale de la préposition

\begin{tabular}{|c|c|c|c|c|c|}
\hline \multirow[b]{2}{*}{ Préposition } & \multicolumn{2}{|c|}{ Anglais } & \multirow[b]{2}{*}{ Préposition } & \multicolumn{2}{|c|}{ Français } \\
\hline & $\%$ & $\mathbf{N}$ & & $\%$ & $\mathbf{N}$ \\
\hline with & 100 & $92 / 92$ & avec & 64 & $25 / 39$ \\
\hline to & 100 & $39 / 39$ & $\grave{a}$ & 0 & $0 / 160$ \\
\hline $\begin{array}{l}\text { about } \\
\text { from }\end{array}$ & $\begin{array}{l}100 \\
71\end{array}$ & $\begin{array}{c}24 / 24 \\
5 / 7\end{array}$ & de & 2 & $2 / 90$ \\
\hline for & 100 & $20 / 20$ & pour & 60 & $3 / 5$ \\
\hline$a t$ & 100 & $18 / 18$ & & & \\
\hline of & 100 & $18 / 18$ & & & \\
\hline in & 88 & $15 / 17$ & $\begin{array}{l}\text { dedans } \\
\text { dans }\end{array}$ & $\begin{array}{c}75 \\
0\end{array}$ & $\begin{array}{l}3 / 4 \\
0 / 9\end{array}$ \\
\hline on & 93 & $14 / 15$ & dessus & 100 & $4 / 4$ \\
\hline into & 100 & $5 / 5$ & & & \\
\hline under & 50 & $1 / 2$ & & & \\
\hline around & 100 & $2 / 2$ & & & \\
\hline through & 100 & $1 / 1$ & à travers de & 100 & $2 / 2$ \\
\hline & & & après & 100 & $2 / 2$ \\
\hline & & & $\begin{array}{l}\text { Inconnue } \\
\text { (absorbée) }\end{array}$ & 0 & $0 / 22$ \\
\hline & & & Autre & 0 & $0 / 3$ \\
\hline Total & 97 & $254 / 260$ & Total & 12 & $41 / 340$ \\
\hline
\end{tabular}

Par ailleurs, le Tableau 10 démontre que l'échouage en français dépend largement du « poids sémantique » de la préposition. Comme on l'a vu, il est à toutes fins pratiques impossible avec les prépositions faibles. En anglais, par contre, l'échouage n'est sensible ni au poids sémantique (voir l'exemple en 13), ni à l'identité lexicale, étant tout aussi fréquent peu importe la préposition impliquée.

Tableau 10. Taux d'échouage dans les langues en contact selon le poids sémantique de la préposition

\begin{tabular}{|l|c|c|c|c|}
\hline & \multicolumn{2}{|c|}{ Anglais } & \multicolumn{2}{c|}{ Français } \\
\hline Poids sémantique & $\%$ & $\mathbf{N}$ & $\%$ & $\mathbf{N}$ \\
\hline Fort & 99 & $68 / 69$ & 50 & $39 / 78$ \\
\hline Faible & 97 & $186 / 254$ & 1 & $2 / 262$ \\
\hline Total & 98 & $254 / 260$ & 12 & $41 / 340$ \\
\hline
\end{tabular}


Ces différences entre la langue donneuse et la langue receveuse dans le domaine des prépositions ne sont que renforcées par un examen de la façon dont les francophones s'en servent lorsqu'ils parlent anglais (Poplack, Zentz et Dion 2012). Toutes leurs prépositions anglaises sont échouées, faisant exactement écho à la répartition manifestée par les anglo-canadiens monolingues (Tableau 7). Aucune ne se voit ni antéposée ni absorbée, en contraste marqué avec le traitement que réservent ces mêmes locuteurs à leurs prépositions lorsqu'ils parlent français (Tableau 1). Dans cette langue, ils n'échouent que la petite cohorte de prépositions fortes, et ce, rarement (12\%), et selon des conditions très précises décrites ci-dessus. Nous concluons qu'ils possèdent deux grammaires indépendantes du placement de la préposition, une pour le français, et une autre pour l'anglais. Voilà encore une preuve éclatante que les prépositions de fin de syntagme en français ne sont pas le produit d'une stratégie anglaise.

Pris ensemble, ces faits excluent à toutes fins pratiques la convergence avec l'anglais comme explication de l'existence des prépositions de fin de syntagme en français. Quelle en est donc l'origine? Le fait que cette variante soit si bien intégrée au sein de la grammaire du placement de la préposition de la langue receveuse (Tableau 6), plutôt que surimposée sur elle (ce à quoi on pourrait s'attendre dans le cas du transfert d'une structure étrangère), suggère une possible motivation interne. Dans la section suivante, en conformité avec le critère $4^{\circ}$ de Poplack et Levey (2011), nous vérifierons donc si une tournure purement française, impliquant elle aussi des prépositions sans régime, sert de modèle pour l'échouage dans les relatives.

\section{Comparaison avec un modèle de souche française : les prépositions orphelines}

Les prépositions qui se trouvent dans cette construction, illustrée en (15), sont parfois appelées « intransitives » (Vinet, 1979, 1984) ou « orphelines » (ORPH. ; Barbaud [1998] ; Roberge [1998] et ZribiHertz [1984] entre autres).

(15) Lui avait trouvé ce charbon-là, puis il se chauffait avec [ ] [ORPH.j. (OH.082.1431)

Leur attestation en français hexagonal remonte au premier dictionnaire de l'Académie française (1694), sinon avant, et ce, sans aucune marque d'usage. Bien qu'il y ait eu un certain flottement en ce qui concerne leur acceptabilité prescriptive à travers le temps (cf. Féraud [1787] et Bescherelle, Bescherelle et de Gaux [1854], par exemple) ${ }^{10}$, les prépositions orphelines sont amplement attestées en français contemporain, comme l'illustre le passage tiré du Bon usage donné en $(16)^{11}$.

10. Nous remercions l'évaluatrice ou l'évaluateur anonyme d'avoir attiré notre attention sur ces précisions historiques.

11. Zribi-Hertz (1984) récapitule la position normative en observant qu'elles sont considérées comme standard lorsque le régime implicite a un référent [-humain], comme en (15); ce n'est que lorsque le régime est [+humain] (20) que les prépositions orphelines tendent à être vues d'un mauvais œil (v. entre autres Grevisse et Goose [2016]). 
(16) Avec les prépositions après, avant, contre, depuis, derrière, devant, l'omission du régime appartient à l'usage le plus général. (Grevisse et Goosse, 2016 : 1440)

Elles ne se produisent pas dans les subordonnées relatives, mais elles sont (théoriquement) admissibles dans n'importe quel complexe verbal transitif à l'indicatif, pourvu qu'il contienne un complément prépositionnel ayant déjà été introduit dans le discours, que ce dernier soit explicite (17a) ou interprétable à partir du contexte plus large $(17 b)^{12}$.

(17) a. Parce que mon père, je me suis tout le temps bien arrangé avec [ ] [ORPH.] $(\mathrm{OH} .002 .1251)$

b. Il faut pâtir pour [ ] [ORPH.]. (OH.071.1644)

Pour la plupart des prépositions, la variante orpheline est en concurrence avec une variante pronominale clitique (CLI.), comme en (18), et avec une construction composée d'une préposition suivie d'un complément pronominal (PRO.), comme en (19) 13 .

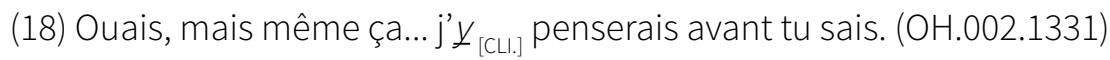

(19) Non, on chantait pas avec ça [Pro.] $(\mathrm{OH} .013 .792)$

Les prépositions orphelines se distinguent donc de celles que nous avons appelées échouées non seulement au niveau des contextes dans lesquels elles apparaissent, mais aussi quant aux variantes avec lesquelles elles sont en concurrence. Elles ne sont donc pas interchangeables avec les prépositions sans régime dans les subordonnées relatives. Mais puisque les variétés du français qui sont à l'abri du contact fournissent ce modèle pour les prépositions en fin de syntagme, il faut les tenir en compte si on veut déterminer la trajectoire par laquelle l'échouage est apparu dans les propositions relatives : est-ce par le biais de la convergence avec l'anglais ou par extension analogique d'un trait purement français? Nous établirons d'abord la grammaire qui donne lieu à ces prépositions orphelines, telle que représentée par le conditionnement quantitatif du choix de la variante, et nous la comparerons ensuite à celle de l'échouage. Si les deux phénomènes obéissaient aux mêmes contraintes linguistiques, cela viendrait appuyer l'analyse selon laquelle le placement de la préposition à la fin du syntagme dans les subordonnées relatives serait une extension des prépositions orphelines à un nouveau contexte. Un tel développement serait motivé par des forces internes plutôt que par le contact. De toute façon, les prépositions orphelines ne sont généralement pas permises avec les équivalents anglais des prépositions françaises pertinentes, tel qu'illustré par la traduction en (20).

12. La préposition orpheline en (17b) réfère à la « bêtise » qu’a faite cette locutrice de ne pas avoir mis sa fille dans une école anglophone.

13. Une quatrième variante, clitique + préposition + complément pronominal, comme en (ii), a été codée dans un premier temps, mais s'est finalement avérée trop rare pour être analysée quantitativement (Zentz, 2006).

(ii) Anglais bien, je vas lui parler à elle, puis lui- lui astheure français, puis il comprend. (OH.013.2108) 
(20) Ça va faire sept ans je reste avec [ ] [ORPH.] $(\mathrm{OH} .090 .1170)$

*'It will be seven years that l'm living with [ ].'

Le Tableau 11, qui montre la répartition des trois variantes principales dans le contexte variable des prépositions orphelines (les complexes verbaux transitifs à l'indicatif contenant un complément prépositionnel ; $N$ = 1644), montre que malgré leur origine française (16), elles ne se produisent que dans 10 \% des énoncés admissibles. Rappelons que c'est sensiblement le même faible taux qu'affichent les prépositions nues en subordonnée relative (Tableau 1). Il est donc clair que la préposition de fin de syntagme, qu'elle soit orpheline ou échouée, est la variante minoritaire.

Tableau 11. Répartition globale des stratégies de placement de la préposition dans le contexte variable des prépositions orphelines

\begin{tabular}{|l|c|c|}
\hline Variantes & \% & N \\
\hline Préposition orpheline & 10 & 160 \\
\hline Clitique & 44 & 720 \\
\hline Complément pronominal & 46 & 764 \\
\hline Total & 100 & 1644 \\
\hline
\end{tabular}

L'analyse multivariée (Tableau 12) révèle que parmi tous les facteurs qui auraient pu affecter le choix d'une préposition orpheline (à la place de l'un de ses concurrents plus fréquents), seuls deux semblent jouer un rôle statistiquement significatif. L'effet de loin le plus important (à en juger par sa portée de 85) est exercé par le poids sémantique de la préposition : à deux exceptions près, les prépositions faibles ne sont jamais orphelines, un résultat qui correspond à la fois à l'usage prescrit (16) et aux observations antérieures de l'usage réel (Barbaud, 1998 ; Roberge, 1998 ; Zribi-Hertz, 1984). Seules leurs homologues sémantiquement riches, que nous avons étiquetées «fortes », apparaissent de façon régulière comme orphelines. Il s'agit là d'exactement le même effet que nous avons décelé pour les prépositions échouées. 
Tableau 12. Analyse multivariée de la contribution de facteurs au choix des prépositions orphelines (seuls les facteurs statistiquement significatifs sont montrés)

\begin{tabular}{|c|c|c|c|}
\hline N total d'occurrences: & \multicolumn{3}{|c|}{$160 / 1643$} \\
\hline \multirow[t]{2}{*}{ Tendance globale: } & \multicolumn{3}{|c|}{.09} \\
\hline & Probabilité & $\%$ & $\mathbf{N}$ \\
\hline \multicolumn{4}{|c|}{ POIDS SÉMANTIQUE DE LA PRÉPOSITION } \\
\hline Forte & .99 & 13 & $158 / 446$ \\
\hline Faible & .16 & .2 & $2 / 1197$ \\
\hline Portée & 83 & & \\
\hline \multicolumn{4}{|l|}{ TYPE DE COMPLÉMENT } \\
\hline Obligatoire & .57 & 7 & $65 / 886$ \\
\hline Facultatif & .42 & 13 & $95 / 758$ \\
\hline Portée & 15 & & \\
\hline
\end{tabular}

Par ailleurs, en regardant de plus près (Tableau 13), on constate qu'ici aussi, l'étiquette de poids sémantique masque un effet lexical idiosyncrasique : à et de représentent 99,7\% des prépositions codées comme «faibles », et tout comme leur comportement dans les prépositions relatives, elles ne sont jamais orphelines. De même, quatre prépositions constituent près de deux tiers de celles qui ont été codées comme «fortes » : dedans, pour, dessus et surtout, avec; celles-ci sont plutôt susceptibles d'être orphelines. Il s'agit bien sûr des mêmes prépositions qui représentent le gros (67\%) de l'échouage dans les subordonnées relatives. Qui plus est, seules quelques-unes des 52 prépositions considérées comme admissibles à être orphelines (Zribi-Hertz, 1984) sont effectivement employées de la sorte dans les données de l'usage. Remarquablement, ce sont les mêmes quelques prépositions qui tendent à être échouées dans les subordonnées relatives (Tableau 2). Nous concluons que le meilleur prédicteur de la propension d'une préposition à paraître sans régime dans les deux contextes que nous avons étudiés est son identité lexicale.

En résumé, nous venons d'examiner un processus de souche française qui produit des prépositions de fin de syntagme, et nous avons trouvé, d'une part, qu'il est contraint par un effet lexical écrasant, et de l'autre, qu'il n'est permis, en général, qu'avec un petit ensemble de prépositions fortes fréquentes. Lorsque nous le comparons au candidat à la convergence, nous trouvons des parallèles incontestables : l'échouage se produit à un taux aussi faible que les prépositions orphelines, et se limite essentiellement au même nombre restreint de prépositions lexicales. La seule explication raisonnable pour ces parallèles - qui sont bien trop détaillés pour être dus au hasard - est que le phénomène de la préposition échouée dans les subordonnées relatives est le résultat d'une extension du processus purement français qui produit des prépositions orphelines. 
Tableau 13. Taux d'occurrence des prépositions orphelines et échouées selon l'identité lexicale et le poids sémantique de la préposition

\begin{tabular}{|c|c|c|c|c|c|c|c|c|}
\hline \multirow[b]{4}{*}{ Identité lexicale } & \multicolumn{8}{|c|}{ Poids sémantique } \\
\hline & \multicolumn{4}{|c|}{ Faible } & \multicolumn{4}{|c|}{ Fort } \\
\hline & \multicolumn{2}{|c|}{ PRÉP. ORPH. } & \multicolumn{2}{|c|}{ PRÉP. ÉCH. } & \multicolumn{2}{|c|}{ PRÉP. ORPH. } & \multicolumn{2}{|c|}{ PRÉP. ÉCH. } \\
\hline & $\%$ & $\mathbf{N}$ & $\%$ & $\mathbf{N}$ & $\%$ & $\mathbf{N}$ & $\%$ & $\mathbf{N}$ \\
\hline$\grave{a}$ & 0 & $2 / 928$ & 0 & $0 / 159$ & 0 & $0 / 15$ & 0 & $0 / 1$ \\
\hline de & 0 & $0 / 266$ & 2 & $2 / 88$ & 0 & $0 / 8$ & 0 & $0 / 2$ \\
\hline avec & - & - & - & - & 40 & $47 / 117$ & 64 & $25 / 39$ \\
\hline dedans & 0 & $0 / 1$ & - & - & 31 & $20 / 65$ & 75 & $3 / 4$ \\
\hline pour & 0 & $0 / 1$ & - & - & 46 & $26 / 57$ & 60 & $3 / 5$ \\
\hline dessus & - & - & - & - & 45 & $23 / 51$ & 100 & $4 / 4$ \\
\hline Autre & 0 & $0 / 2$ & 0 & $0 / 15$ & 25 & $42 / 133$ & 17 & $4 / 23$ \\
\hline Total & 0 & $2 / 1198$ & 1 & $2 / 262$ & 35 & $158 / 446$ & 50 & $39 / 78$ \\
\hline
\end{tabular}

\section{Comparaison avec un stade antérieur au contact}

Afin de satisfaire aux critères $1^{\circ}$ et $2^{\circ}$ de Poplack et Levey (2011) nécessaires pour légitimer l'hypothèse de la convergence (section 1.1), il nous reste à établir si l'échouage est le résultat d'un changement. Le cas échéant, nous ne nous attendrions pas à le trouver dans un stade antérieur du français. Comme nous l'avons précisé ci-dessus, notre objet d'étude est la langue parlée - lieu privilégié pour la convergence. Pour que la comparaison soit valable, le stade antérieur doit lui aussi impliquer la langue parlée. Cette obligation limite nécessairement la portée temporelle de notre analyse (les enregistrements sonores étant peu courants, voire inexistants avant la deuxième moitié du XIXe siècle). Heureusement, cependant, nous pouvons exploiter les données renfermées dans le corpus des Récits du français québécois d'autrefois (Poplack et St-Amand, 2009). Elles reflètent le parler informel de Québécois isolés en milieu rural, nés dans la deuxième moitié du XIXe siècle, c'est-à-dire loin - temporellement et géographiquement - du contact intense actuel avec l'anglais. Nous avons systématiquement cherché dans ce corpus les occurrences de prépositions en fin de syntagme, et en avons identifié 102 qui figurent dans le(s) contexte(s) variable(s) étudié(s) ici, presque toutes des orphelines. La vaste majorité (82 \%) consistait en avec, comme en (21), préfigurant ainsi la disproportion observée dans le français d'Ottawa-Hull du XXe siècle. Les prépositions orphelines étaient déjà clairement robustes dans le français québécois parlé au XIXe siècle.

(21) a. Prends cet âne-là, puis va-t'en chez vous avec [ ] [ORPH.] $($ RFQ.014.30)

b. Puis elle le pogne puis elle s'en va dans la forêt avec [ ] [ORPH.] $_{\text {(RFQ.023.1879) }}$ 
Or, le candidat à la convergence, quoique relativement rare dans ces données ( $N=5)$, était lui aussi déjà attesté, tel qu'illustré en $(22)^{14}$.

(22) a. Elle se nourrit aux crabes. Une quantité de crabes que la morue mange, qu'elle - qu'elle se nourrit avec ${ }_{[\mathrm{E} C \mathrm{CH}]}$ (RFQ.043.1057)

b. Elle leur a emporté trois plombs qu'ils pêchent avec ${ }_{[\mathrm{E} C H]}$. RFQ.043.1420)

Ce résultat ne devrait pas surprendre ; la préposition échouée était déjà attestée dans la Grammaire des fautes de Frei ([1929/1971 : 187] ; voir aussi Bauche [1920]), qui malgré son titre, s’identifie comme une description linguistique du parler populaire spontané du français (hexagonal) (23)15.

(23) La jeune fille qu'il doit se marier avec ${ }_{[\mathrm{EECH}]}$; Le pont qu'il est passé dessus ${ }_{[\mathrm{E} C H]}$; La caisse que c'est mis dedans ${ }_{[\mathrm{E} C \mathrm{CH}]}$; Je n'ai pas reçu le colis qu'elles étaient dedans ${ }_{[\mathrm{E} C \mathrm{CH}]}$.

Le fait que dans le français parlé du XIXe siècle elle ne se produit qu'au moyen d'avec (la préposition échouée la plus fréquente dans le français du XXe siècle [Tableau 3], de même que celle qui est le plus souvent orpheline) témoigne à nouveau de la relation synergique entre les prépositions échouées et orphelines.

Finalement, il serait négligent de notre part de ne pas mentionner que les prépositions de fin de syntagme apparaissent également en subordonnée relative dans une variété contemporaine qui est à l'abri du contact, quoiqu'apparemment moins fréquemment. II s'agit du français hexagonal, que l'on ne considère généralement pas enclin à la convergence avec l'anglais. Le chanteur Renaud en offre un bon exemple dans sa chanson Ma gonzesse.

\section{Discussion et conclusions}

L'objectif de la recherche rapportée ici était de proposer et d'appliquer une méthode empirique pour établir, par la concrétisation et mise à l'épreuve d'hypothèses, l'effet qu'une langue donneuse peut exercer sur une langue receveuse, surtout quand celle-ci revêt un statut minoritaire. Nous avons illustré l'utilité de cette méthode, fondée sur les préceptes de la sociolinguistique variationniste, par le cas des prépositions sans régime, trait non standard stéréotypé associé à l'influence de l'anglais

14. La disproportion reflète, bien qu'à un degré moindre, la rareté relative des prépositions échouées vis-à-vis les orphelines déjà notée pour le XXe siècle ( $26 \%$; 41/160), vraisemblablement en raison du petit nombre de propositions relatives déterminatives, le seul contexte qui admet ces dernières.

15. Une évaluatrice ou un évaluateur anonyme remarque que puisque Bauche et Frei sont tous les deux nés au milieu du XIX siècle, il y a de fortes chances que les prépositions échouées, tout comme leurs contreparties orphelines et absorbées, étaient aussi déjà courantes avant cette époque, du moins dans des variétés populaires de français hexagonal (et canadien). La question du moment précis de l'entrée de ces premiers en français, quoiqu'importante, dépasse le cadre de cette étude. Pour nos fins actuelles, il suffit d'observer qu'il était bien antérieur au contact de l'anglais et du français en terre canadienne. 
langue majoritaire sur le français nord-américain. Conscientes que le changement, qu'il soit de nature convergente ou autre, ne naît et ne s'étend que par le biais d'interactions régulières, nous sommes allées droit à la source, en privilégiant une communauté bilingue florissante comme lieu de recherche, et les échanges spontanées de ses membres comme données.

Ce travail part du principe selon lequel, pour déceler un changement, il faut aller au-delà de l'assimilation habituelle de formes supposément déviantes à des équivalents superficiellement semblables dans une langue donneuse. Ceci implique la reconnaissance, d'abord et avant tout, du lien inexorable entre le changement en cours et la variabilité linguistique, ainsi que de la concurrence entraînée par cette variabilité parmi les formes variantes. Plutôt que de nous concentrer uniquement sur le candidat à la convergence, ceci nous oblige à le situer par rapport au système linguistique plus large duquel il fait partie. Comme nous l'avons démontré, le choix de la variante est sujet à un ensemble de conditions qu'il est possible (moyennant une méthodologie adéquate) de découvrir ; celles-ci constituent la structure de la variabilité. Une fois établie, cette structure peut être mise au service de comparaisons entre diverses variétés-repère, qui à leur tour servent de diagnostics de la stabilité ou du changement. En nous servant du cadre théorique variationniste comparatif (Poplack et Meechan, 1998 ; Poplack et Tagliamonte, 2001 ; Tagliamonte, 2002), nous avons donc comparé les contraintes variables sur le choix du candidat à la convergence $1^{\circ}$ ) dans les langues donneuse et receveuse ; $2^{\circ}$ ) avant et après le contact avec la langue receveuse ; $3^{\circ}$ ) avec une variété cible de la langue donneuse; et $4^{\circ}$ ) le plus révélateur, avec les contraintes qui gouvernent les variantes avec lesquelles il coexiste dans le système de la langue receveuse. Nous avons aussi évalué la possibilité de l'existence d'un modèle interne au français pour les prépositions de fin de syntagme grâce à la comparaison détaillée avec une tournure qui existait déjà dans la langue au départ.

Nos analyses ont révélé plusieurs sources de preuves indépendantes contrecarrant une interprétation de convergence. Le premier élément important est la découverte que les conditions qui donnent lieu aux prépositions nues dans les subordonnées relatives françaises ne sont nulles autres que celles qui produisent les prépositions orphelines parfaitement acceptables en français normé dans d'autres contextes. Ensuite, en situant les prépositions apparaissant à la fin du syntagme par rapport aux autres possibilités de placement de la préposition dans le contexte variable constitué par les subordonnées relatives, nous avons appris que plutôt que d'empiéter sur ce système serré, comme le ferait un changement externe motivé par le contact, les prépositions nues y jouent en fait un rôle spécialisé. De plus, la comparaison avec un état de la langue antérieur au contact révèle que les prépositions nues étaient non seulement déjà présentes dans le contexte crucial des subordonnées relatives, mais (à en juger par les données disponibles) elles observaient le même schéma de conditionnement constaté dans les données contemporaines. Par ailleurs, la comparaison avec la source présumée démontre qu'aucune des contraintes régissant les prépositions sans régime en français n'est en vigueur en anglais, qui, lui, échoue librement - en effet, presque catégoriquement ses prépositions dans toute une gamme de contextes. Ces contextes diffèrent à leur tour de ceux qui affichent les prépositions nues - tant orphelines qu'échouées - en français. Finalement, notre 
comparaison des stratégies de placement chez les mêmes locuteurs dans les deux langues (section 5.1) montre qu'en dépit du contact intense au niveau communautaire, les individus bilingues maintiennent deux grammaires séparées, une pour l'anglais, et l'autre pour le français.

À la lumière des résultats présentés ici, nous sommes pour le moins frappées par l'incongruité de la quantité d'attention (négative!) consacrée à l'échouage comme emblème du changement induit par le contact en français canadien, alors qu'il s'agit en réalité d'un phénomène mineur, tant au niveau des contextes où il peut se produire que de ceux où il se produit effectivement. Conclure que les prépositions sans régime sont le fruit de la convergence peut paraitre logique si l'on suppose, comme le font apparemment plusieurs, qu'une ressemblance de surface (ici l'absence de régime chez certaines prépositions) équivaut à une ressemblance structurelle. Or, lorsque nous étudions les conditions qui contraignent la sélection variable de ce candidat à la convergence, nous apprenons que les parallèles de surface recèlent une divergence importante de structure.

La primauté de la langue orale dans le déclenchement et la diffusion du changement est irréfutable. La marque du parler est sa variabilité inhérente, qui est contrainte par des facteurs qui ne peuvent être découverts que par une analyse systématique quantitative, comme celle que nous avons exposée ici. Lorsque nous nous en servons pour situer le candidat au changement par rapport à tous les contextes pertinents dans lesquels il évolue, son rôle devient clair, tout comme la trajectoire par laquelle il s'est développé, à savoir, conjointement aux prépositions orphelines françaises, et non par voie de l'emprunt à l'anglais.

Nous ne prétendons pas que le contact entre langues est incapable de provoquer la convergence grammaticale. Mais une telle issue ne peut pas être tenue pour acquise, même quand les circonstances y sont très favorables, comme c'est le cas pour la situation ciblée dans cette étude. Pour prouver que la convergence a eu lieu, il faut une méthodologie scientifique comme celle que nous avons présentée ci-dessus (voir aussi Poplack, 1993, sous presse; Poplack et Levey, 2011). Au moment où nous écrivons ce chapitre, de telles preuves sont peu nombreuses. En attendant, à la lumière des résultats que nous avons relevés ici, ainsi que ceux d'autres candidats à la convergence grammaticale (p. ex. Leroux et Jarmasz, 2006 ; Otheguy, Zentella et Livert, 2007 ; Poplack, 1997 ; Torres Cacoullos et Travis, 2018) et d'autres types d'anglicismes (à savoir l'emprunt lexical et l'alternance de code), notre réponse à la question « les anglicismes mènent-ils inexorablement à la convergence grammaticale? » est un non retentissant. 


\section{Références}

Ambrose, Jeanne (1987), Syntaxe comparative du français et de l'anglais, Potomac, MD, Scripta Humanistica.

Appel, René et Pieter Muysken (1987), Language Contact and Bilingualism, Londres, Edward Arnold.

Backus, Ad (2004), «Convergence as a mechanism of language change », Bilingualism: Language and Cognition, vol. 7, n² 2, p. 179-181.

Backus, Ad (2005), «Code-switching and language change: One thing leads to another? », International Journal of Bilingualism, vol. 9, n³/4, p. 307-340.

Barbaud, Philippe (1998), « Dissidence du français et évolution dialectale », Revue québécoise de linguistique, vol. 26, n², p. 107-128.

Bauche, Henri (1920), Le langage populaire : grammaire, syntaxe et dictionnaire du français tel qu'on le parle dans le peuple de Paris, avec tous les termes d'argot usuel, Paris, Payot.

Bescherelle, Louis Nicolas, Henri-Honoré Bescherelle et Litais de Gaux (1854), Grammaire nationale, Paris, Garnier frères.

Bouchard, Denis (1982), «Les constructions relatives en français vernaculaire et en français standard : Étude d'un paramètre », dans Claire Lefebvre (dir.), La syntaxe comparée du français standard et populaire: Approches formelle et fonctionnelle, Québec, Éditeur officiel du Québec, vol. 1, p. 103-133.

Budzhak-Jones, Svitlana (1998), Single-Word Incorporations in Ukrainian-English Bilingual Discourse: Little Things Mean a Lot, thèse de doctorat, Ottawa, Université d'Ottawa.

Delisle, Jean (1998), « Les anglicismes insidieux », L’Actualité terminologique, vol. 31, n³, p. 10-14.

Féraud, Jean-François (1787), Dictionnaire critique de la langue française, Jean Mossy.

Flikeid, Karen (1989), "'Moitié anglais, moitié français'? Emprunts et alternance de langues dans les communautés acadiennes de la Nouvelle-Écosse », Revue québécoise de linguistique théorique et appliquée, vol. 8, n² 2, p. 177-228.

Frei, Henri (1971), La grammaire des fautes, Genève, Slatkine Reprints. [1éd., 1929.]

Grevisse, Maurice et André Goosse (2016), Le bon usage : Grammaire française, 16e éd., Louvain-LaNeuve, De Boeck Supérieur, Duculot. [1 $1^{\text {re }}$ é., 1936.]

Harris, Alice et Lyle Campbell (1995), Historical Syntax in Cross-Linguistic Perspective, Cambridge, Cambridge University Press.

Heine, Bernd et Tania Kuteva (2005), Language Contact and Grammatical Change, Cambridge, Cambridge University Press. 
King, Ruth (2000), The Lexical Basis of Grammatical Borrowing: A Prince Edward Island French Case Study, Philadelphia, John Benjamins.

King, Ruth et Yves Roberge (1990), «Preposition stranding in Prince Edward Island French », Probus, vol. 2, n³, p. 351-369.

Koster, Jan (1978), Locality Principles in Syntax, Dordrecht, Foris.

Labov, William (1969), "Contraction, deletion and inherent variability of the English copula », Language, vol. 45, n 4, p. 715-762.

Leroux, Martine et Lidia-Gabriela Jarmasz (2006), « A study about nothing: Null subjects as a diagnostic of convergence between English and French », Penn Working Papers in Linguistics, vol. 12, n 2 (Selected Papers from NWAV 34), p. 1-14.

Moinzadeh, Ahmad (1999), «Bilingual phenomena: Towards the fateful triangle of language mixture », Cahiers linguistiques d'Ottawa, vol. 27, p. 31-63.

Mustafawi, Eiman (2002), « Lone English-origin nouns in Arabic: Codeswitches or borrowings? », dans Sophie Burelle et Stanca Somesfalean (dir.), Proceedings of the 2002 Annual Conference of the Canadian Linguistic Association, Université du Québec à Montréal, p. 219-231.

Otheguy, Ricardo, Ana Celia Zentella et David Livert (2007), « Language and dialect contact in Spanish in New York: Toward the formation of a speech community », Language, vol. 83, n 4, p. 770-802.

Poplack, Shana (1980), "'Sometimes l'll start a sentence in Spanish y termino en español' : Toward a typology of code-switching », Linguistics, vol. 18, nºs 7-8, p. 581-618.

Poplack, Shana (1985), "Contrasting patterns of code-switching in two communities », dans Henry J. Warkentyne (dir.), Methods V: Papers from the V International Conference on Methods in Dialectology, Victoria, Colombie-Britannique, University of Victoria, p. 363-385.

Poplack, Shana (1989a), « The care and handling of a mega-corpus: The Ottawa-Hull French project», dans Ralph Fasold et Deborah Schiffrin (dir.), Language Change and Variation, Amsterdam, Benjamins, p. 411-451.

Poplack, Shana (1989b), «Statut de langue et accommodation langagière le long d'une frontière linguistique », dans Raymond Mougeon et Édouard Beniak (dir.), Le français canadien parlé hors Québec: Aperçu sociolinguistique, Québec, Presses de l'Université Laval, p. 127-151.

Poplack, Shana (1993), «Variation theory and language contact», dans Dennis Preston (dir.), American Dialect Research: An Anthology Celebrating the 100 ${ }^{\text {th }}$ Anniversary of the American Dialect Society, Amsterdam, Benjamins, p. 251-286.

Poplack, Shana (1997), «The sociolinguistic dynamics of apparent convergence », dans Gregory Guy, Crawford Feagin, Deborah Schiffrin et John Baugh (dir.), Towards a Social Science of Language: Papers in Honor of William Labov, Amsterdam, Benjamins, p. 285-309.

Poplack, Shana (2015), « Code-switching (Linguistic) », dans International Encyclopedia of the Social and Behavioral Sciences, 2 éd., Amsterdam, Elsevier Science Ltd, p. 918-925. [1'e éd., 2001.] 
Poplack, Shana (2017), «L'anglicisme chez nous: Une perspective sociolinguistique », dans Les anglicismes : Des emprunts à intérêt variable? Recueil des actes: Colloque du réseau des Organismes francophones de politique et d'aménagement linguistiques (OPALE), 19 et 20 octobre 2016, Montréal, Publications de l'Office québécois de la langue française, p. 375-403.

Poplack, Shana (2018), Borrowing: Loanwords in the Speech Community and in the Grammar, Oxford, Oxford University Press.

Poplack, Shana (sous presse), «A variationist perspective on language contact », dans Evangelia Adamou et Yaron Matras (dir.), Routledge Handbook of Language Contact, Routledge.

Poplack, Shana et Nathalie Dion (2012), « Myths and facts about loanword development », Language Variation and Change, vol. 24, n³, p. 279-315.

Poplack, Shana et Marjory Meechan (1998), «Introduction: How languages fit together in codemixing », International Journal of Bilingualism, vol. 2, n² 2, p. 127-138.

Poplack, Shana et Anne St-Amand (2009), "Les Récits du français québécois d'autrefois : Reflet du parler vernaculaire du XIX siècle », Revue canadienne de linguistique, vol. 54, n 3, p. 511-546.

Poplack, Shana et Sali Tagliamonte (2001), African American English in the Diaspora, Oxford, Blackwell Publishers.

Poplack, Shana, James A. Walker et Rebecca Malcolmson (2006), "An English 'like no other'?: Language contact and change in Quebec », Revue canadienne de linguistique, vol. 51, nos 2-3, p. 185-213.

Poplack, Shana, Lauren Zentz et Nathalie Dion (2012), « Phrase-final prepositions in Quebec French: An empirical study of contact, code-switching and resistance to convergence », Bilingualism : Language and Cognition, vol. 15, n² 2, p. 203-225.

Radio-Canada (2018), Le franglais n'affaiblit pas le français, selon une étude de l'Université d'Ottawa, disponible sur https://ici.radio-canada.ca/nouvelle/1082833/franglais-affaiblit-francais-etude-universite-ottawa-shana-poplack-bilingue-anglais. [Page consultée le 8 février 2018.]

Roberge, Yves (1998), «Les prépositions orphelines dans diverses variétés de français d'Amérique du Nord », dans Patrice Brasseur (dir.), Français d'Amérique : Variation, créolisation, normalisation, Avignon, Centre d'études canadiennes, p. 49-60.

Roberge, Yves et Nicole Rosen (1999), «Preposition stranding and que-deletion in varieties of North American French », Linguistica Atlantica, vol. 21, p. 153-168.

Roy, Marie-Marthe, Claire Lefebvre et Andrée Régimbald (1982), « Acquisition de la norme et de la structure linguistique des relatives chez deux groupes d'adolescents montréalais », dans Claire Lefebvre (dir.), La syntaxe comparée du français standard et populaire: Approches formelle et fonctionnelle, vol. 2, Québec, Gouvernement du Québec, Office de la langue française. 
Sankoff, David (1988), « Variable rules », dans Ulrich Ammon, Norbert Dittmar et Klaus. J. Mattheier (dir.), Sociolinguistics: An International Handbook of the Science of Language and Society, Berlin, Walter de Gruyter, p. 984-987.

Sankoff, David et Shana Poplack (1981), «A formal grammar for code-switching », Papers in Linguistics, vol. 14, n 1, p. 3-46.

Sankoff, David, Sali Tagliamonte et Eric Smith (2005), «Goldvarb X», Logiciel informatique, Department of Linguistics, University of Toronto, Canada.

Tagliamonte, Sali (2002), " Comparative sociolinguistics », dans Jack Chambers, Peter Trudgill et Natalie Schilling-Estes (dir.), Handbook of Language Variation and Change, Malden et Oxford, Blackwell Publishers, p. 729-763.

Takami, Ken-ichi (1992), Preposition Stranding: From Syntactic to Functional Analyses, New York, Mouton de Gruyter.

Thomason, Sarah G. (2001), Language Contact: An Introduction, Edinburgh, Edinburgh University Press.

Toribio, Almeida Jacqueline (2004), « Convergence as an optimization strategy in bilingual speech: Evidence from code-switching », Bilingualism: Language and Cognition, vol. 7, n² 2, p. 165-173.

Torres Cacoullos, Rena et Catherine Travis (2018), Bilingualism in the Community: Code-switching and Grammars in Contact, Cambridge, Cambridge University Press.

Université d'Ottawa (2018), Divulgâcheur: le "franglais », ce n'est pas si mauvais, disponible sur https://medias.uottawa.ca/nouvelles/divulgacheur-franglais-ce-nest-pas-si-mauvais. [Page consultée le 6 février 2018.]

Vinet, Marie-Thérèse (1979), « Dialect variation and a restrictive theory of grammar: A study of intransitive prepositions in a variety of French », Recherches linguistiques à Montréal, vol. 13, p. 107-125.

Vinet, Marie-Thérèse (1984), «La syntaxe du québécois et les emprunts à l'anglais », Revue de l'association québécoise de linguistique, vol. 3, n³, p. 221-242.

Winford, Donald (2005), «Contact-induced changes: Classifications and processes », Diachronica, vol. 22, n², p. 373-427.

Zentz, Lauren (2006), 'C'estça je travaille dessus': Orphaned prepositions and relativization in Canadian French, thèse de maitrise, Ottawa, Université d'Ottawa.

Zribi-Hertz, Anne (1984), Orphan Prepositions in French and the Concept of 'Null Pronoun', Bloomington, IN, Indiana University Linguistics Club. 